\title{
Visual search asymmetries in motion and optic flow fields
}

\author{
CONSTANCE S. ROYDEN \\ College of the Holy Cross, Worcester, Massachusetts \\ JEREMY M. WOLFE \\ Brigham and Women's Hospital and Harvard Medical School, Boston, Massachusetts \\ and \\ NICOLE KLEMPEN \\ Brigham and Women's Hospital, Boston, Massachusetts
}

\begin{abstract}
In visual search, items defined by a unique feature are found easily and efficiently. Search for a moving target among stationary distractors is one such efficient search. Search for a stationary target among moving distractors is markedly more difficult. In the experiments reported here, we confirm this finding and further show that searches for a stationary target within a structured flow field are more efficient than searches for stationary targets among distractors moving in random directions. The structured motion fields tested included uniform direction of motion, a radial flow field simulating observer forward motion, and a deformation flow field inconsistent with observer motion. The results using optic flow stimuli were not significantly different from the results obtained with other structured fields of distractors. The results suggest that the local properties of the flow fields rather than global optic flow properties are important for determining the efficiency of search for a stationary target.
\end{abstract}

In a search for a target in the midst of a field of distractors, targets defined by a basic visual feature, such as color or orientation, often appear to "pop out" from the background of distractor items. People locate these items with ease, independent of the number of distractors present. Such searches have been classically labeled as "parallel" (e.g., Treisman \& Gelade, 1980) since it appears that all items can be processed at once. A more theoretically neutral label might be "very efficient" (Wolfe, 1998). In other cases, the target is more difficult to find. Reaction time (RT), the time required to determine if a target is present, increases $20-30 \mathrm{msec}$ with each additional distractor in the field on target present trials and twice that rate, $40-60 \mathrm{msec} / \mathrm{item}$, on target absent trials. These searches have been classically labeled as "serial" since the RTs are consistent with a serial, item-by-item search at a rate of one item every 40-60 msec (Treisman \& Gelade, 1980). Great debate has swirled around the concept of serial search (Moore \& Wolfe, 2000; Townsend, 1990), and again, in an effort to be theoretically neutral, these could be called "inefficient" searches.

We thank Todd Horowitz, Serena Butcher, Chris Pack, and George Alvarez for comments on earlier drafts of this paper. We thank Francis Ho for help collecting the data. This research was supported by grants from the National Eye Institute of the NIH (EY05087) and the Human Frontiers Science Program. Correspondence should be addressed to J. M. Wolfe, Center for Ophthalmic Research, Brigham and Women's Hospital, 221 Longwood Ave., Boston, MA 02115 (e-mail: wolfe@ search. bwh.harvard.edu).
The most efficient visual searches are those in which the target is defined by the presence of a "basic feature," like color, size, or orientation and where the distractors are homogeneous (Duncan \& Humphreys, 1989). There is a limited set of basic features (on the order of a dozen, reviewed in Wolfe, 1998). When examining these basic feature searches, researchers have often found asymmetries in search efficiency between certain pairs of stimuli. A visual search asymmetry refers to the situation in which it is easier to find $A$ as the target among distractors of type B than it is to find $\mathrm{B}$ as a target among distractors of type A (Treisman \& Souther, 1985). For example, in color search, it is easier to find a magenta target among red distractors than it is to find a red target among magenta distractors (Treisman \& Gormican, 1988). Such asymmetries have been found with a variety of basic features, such as gap detection, line convergence, shape (Treisman \& Gormican, 1988), orientation (Foster \& Ward, 1991), orientation in depth (Von Grünau \& Dubé, 1994), and so forth. In the motion domain, Ivry and Cohen (1992) have shown an asymmetry between fast and slow moving stimuli, with subjects' finding a fast moving target among slow distractors much more efficiently than they found a slow target among fast distractors.

As a possible explanation for these search asymmetries, Treisman (Treisman \& Souther, 1985) has suggested that it is easier to detect the presence of a basic feature than it is to locate its absence. Thus, the presence of "blueness" within a magenta target is easy to detect among red distractors, whereas the absence of blueness in the red 
target is more difficult to locate among the magenta distractors. If this were the case, one would expect that for the basic feature of motion, it should be easier to detect a moving target among stationary distractors than to detect a stationary target among moving distractors. Several published reports have demonstrated that it is very easy to find a moving target among stationary distractors (Dick, Ullman, \& Sagi, 1987; McLeod, Driver, \& Crisp, 1988; Nakayama \& Silverman, 1986). Verghese and Pelli (1992) found that a moving target among stationary distractors is found more efficiently than is a stationary target among moving distractors. In Experiment 1, we confirmed and extended this finding by examining whether the type of motion undergone by the distractors affected the efficiency of search.

In Experiment 2, we considered the case of a moving observer. Observer motion produces stimulus motion on the retina. A stationary item in the world may become a moving stimulus on the retina. A moving stimulus can become stationary. Do the rules governing visual search for motion refer to motion of the distal stimulus or of the proximal stimulus? Consider a person who is driving a car with a dead fly on the windshield. The fly is stationary in the retinal image, but moving in the world. Objects along the roadside are stationary in the world, but move on the retina. One can imagine designing a visual system that could discount retinal motion that is due to observer motion. Thus, an object moving in the world would "pop out" even though the retinal images of the distractors are themselves in motion. Similarly, any search asymmetries found between moving and stationary items would be the same whether or not the observer is moving. The idea that observer motion might be discounted pre-attentively is credible, because the "features" used to guide visual search can be fairly highly processed. For example, search based on orientation differences can be efficient when the orientation is defined by second-order stimuli, such as color, texture, motion, or depth differences (Bravo \& Blake, 1990; Cavanagh, Arguin, \& Treisman, 1990; Gurnsey, Humphrey, \& Kapitan, 1992). Thus, preprocessing of optic flow fields could conceivably lead to efficient search for a moving object in the world, independent of observer motion. In Experiment 2, we examined whether search asymmetries for moving and stationary objects in the world remain constant for a moving observer.

\section{General Method}

Stimuli were presented on a Macintosh PowerPC computer that displayed white circles on a black background. The frame rate of each presentation was 30 frames per second. The diameter of each circle was $1.15^{\circ}$. In the first experiment, the circles moved at a constant speed of $1.64 \%$ sec. In the second experiment, the speed of the circles depended on the simulated observer motion. The circles were initially positioned within a $20^{\circ} \times 20^{\circ}$ window. The window was evenly divided into a square grid, with the number of grid elements equal to the number of items in the display. Each circle was positioned randomly within a single square of that grid so that the average density of items was uniform across the display (i.e., one item per square unit in the viewing window). The size of the viewing window was $30^{\circ} \times 30^{\circ}$, so no items moved out of the window during any trial. The subjects viewed the screen from a distance of $57.4 \mathrm{~cm}$ and were free to move their eyes. Set sizes of $4,9,16$, or 25 items were randomly intermixed in a block of trials. The stimuli remained on the screen until the subject made a response or for a maximum of $2.5 \mathrm{sec}$.

The subjects were instructed to begin each trial by pressing the space bar with both hands. A target item was designated before each block of trials. The target was present on $50 \%$ of the trials, and the subject indicated by a keypress whether or not the target was present on each trial. Feedback was given in the form of a beep after each incorrect response. Each subject ran 320 trials [4 set sizes $\times 2$ object conditions (present or absent) $\times 40$ repetitions] per block.

Sixteen subjects participated in Experiment 1 and 21 in Experiment 2. The subjects were between the ages of 18 and 55. All had visual acuity of 20/25 or better and could pass the Ishihara color test. The subjects gave informed consent and were paid for their time. All were naive as to the purposes of the experiment. The order of conditions was randomized across subjects.

\section{EXPERIMENT 1 Moving Versus Stationary Items}

\section{Method}

This experiment extended the experiments of Verghese and Pelli (1992) that tested whether a search asymmetry exists between moving and stationary targets. These are like searches for live flies among dead flies, or vice versa. The subjects searched for either a moving target among stationary distractors (the live-fly condition) or a stationary target among moving distractors (the dead-fly condition). For each of these conditions, we tested three types of motion: uniform, random, or Brownian. In the uniform motion experiment, all of the circles moved in the same direction. The direction was randomly selected for each trial between $0^{\circ}$ and $360^{\circ}$. In the random motion experiment each circle moved in a straight line in a direction chosen at random between $0^{\circ}$ and $360^{\circ}$. Thus, there was no organized pattern of motion in this condition. In brownian motion, each moving item engaged in a two-dimensional random walk, changing direction of motion every $200 \mathrm{msec}$. The brownian motion condition is similar to the condition tested by Verghese and Pelli. The uniform and random motion conditions tested whether a motion field that is more structured over time (random) and/or space (uniform) will increase the efficiency of the search task. In the random and brownian motion experiments, each circle moved independently of the others. The uniform and random motion conditions were identical for the live-fly experiments, since these consisted of the target moving in a randomly chosen direction. Illustrations of these object motions are shown in Figure 1.

\section{Results}

Figure 2 shows the mean RTs, averaged across all 16 subjects. The slopes for each condition are given in Table 1. The main trends of the data are clear. Search for a stationary, "dead" item among moving, "live" items is markedly less efficient than search for a live item among dead distractors [ANOVA: target present trials, $F(1,15)=$ 


\section{Uniform Motion}
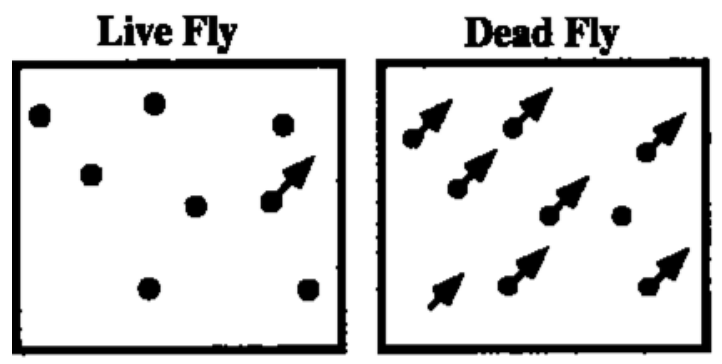

Random Motion
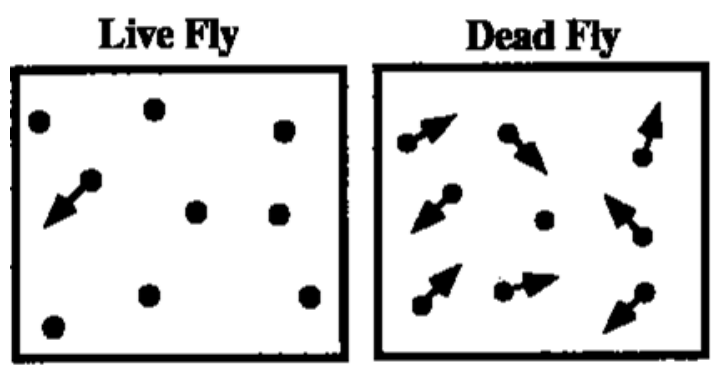

\section{Brownian Motion}
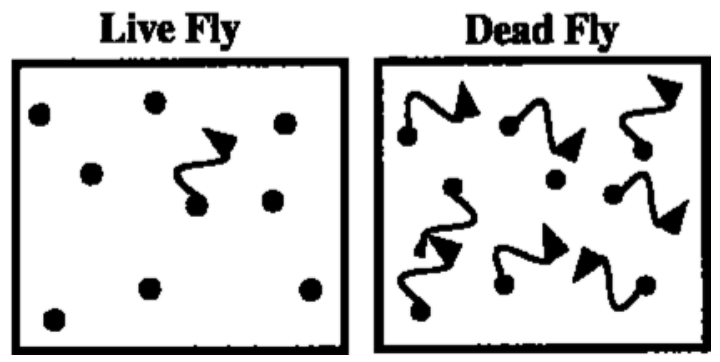

Figure 1. Stimulus conditions for Experiment 1. For the uniform and random motion conditions, arrows indicate the direction of motion of individual circles. For the Brownian motion condition, the arrows indicate a random path of motion for each circle.

$106.4, p<.0001$; target absent trials, $F(1,15)=140.7$, $p<.0001]$. This effect is more marked for the random and brownian conditions but is clearly present in the uniform condition as well (paired $t$ test, $p<.0002$ ). The brownian and random motion conditions produced significantly less efficient search than did the uniform condition (Fisher's PLSD test; $p<.001$ ). The live conditions produced slopes that are not significantly greater than zero (each of six, one-tailed $t$ tests yield $p>.66$ ). Indeed, the slopes of the target absent trials and the slopes of the uniform target present trials are significantly less than zero (all one-tailed $t$ tests, $p<.01$ ), something that is sometimes seen with the most basic of basic feature searches. The greater density of larger set sizes actually speeds search.

The RT data understate the degree of the asymmetry in motion search. Figure 3 shows error rates as a function of set size. Error rates were much higher in the search for a dead target (all paired $t$ tests, $p<.01$ ). The huge "miss" rates in the random and brownian conditions are a side effect of the limited duration of the stimuli. Some subjects could not find dead targets, even after $2.5 \mathrm{sec}$. They were forced to guess and generally assumed that, if they did not see it, it was not there. We should assume, therefore, that the slopes shown in Table 1 are underestimates and that underestimation is most marked in the dead conditions.
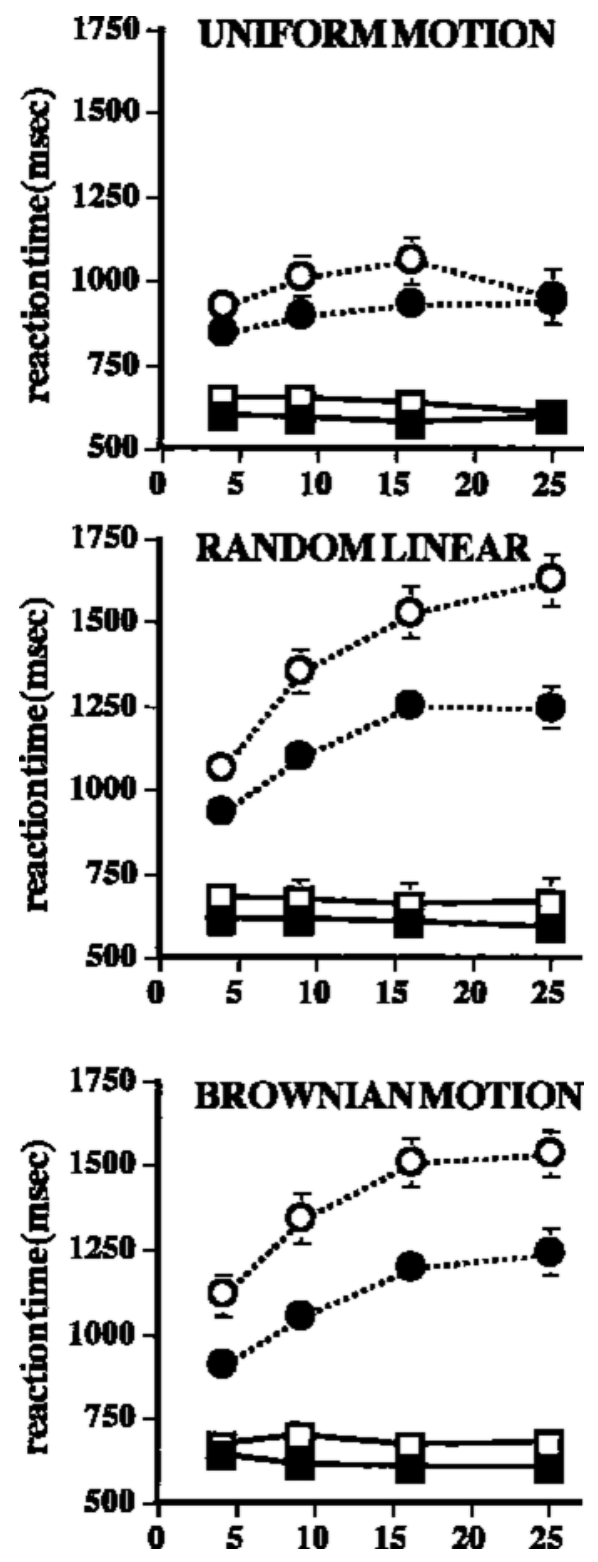

Figure 2. Results of Experiment 1. Each graph shows the RT $\times$ set size functions for the three motion conditions in Experiment 1 averaged across 16 subjects. Circles show the dead-fly conditions, and squares indicate the live-fly conditions. Open symbols represent target absent conditions, and filled symbols represent target present conditions. Error bars (where visible) are $\pm 1 S E$. 
Table 1

Slopes for Each of the RT $\times$ Set Size Functions for Experiment 1

\begin{tabular}{|c|c|c|c|c|c|c|}
\hline & \multicolumn{2}{|c|}{ Uniform Motion } & \multicolumn{2}{|c|}{ Random Linear Motion } & \multicolumn{2}{|c|}{$\underline{\text { Brownian Motion }}$} \\
\hline & Live & Dead & Live & Dead & Live & Dead \\
\hline Present & -0.2 & 4.2 & -1.2 & 14.7 & -1.6 & 15.9 \\
\hline Absent & -2 & 0.9 & -0.6 & 25.4 & -0.2 & 19.3 \\
\hline
\end{tabular}

Note-The slopes are given in milliseconds per item. (See Figure 2 for graphs of the slopes.)

\section{Discussion}

Experiment 1 confirmed that search for a stationary item among moving items is harder than search for a moving item among stationary items. If we accept Treisman's (Treisman \& Souther, 1985) conjecture that it is easier to find the presence of a basic feature than it is its absence, then these results suggest that motion is a feature and stationary is its absence, rather than its having featural status in its own right. It seems unlikely that this effect is an artifact of our particular choice of dot sizes or stimulus velocities. Nor is it an artifact of a few, very error prone subjects. If we reanalyze the results using only the data from the half of the subjects with the lowest error rates, we would obtain the same pattern of results seen in the full set of subjects.

In addition to the asymmetry between live- and deadfly conditions, we also see a difference in the efficiency of search between the random motion conditions and the uniform condition. This supports the observation that search efficiency declines as distractor heterogeneity increases (Duncan \& Humphreys, 1989). Rosenholtz (2001) has offered a different account of asymmetries that might explain this result. She notes that search tasks involving random motion are inherently asymmetrical since, in the live-fly condition, the distractors occupy a single locus in velocity space whereas, in the dead-fly condition, the distractors occupy multiple locations. Worse, the area described by those distractor locations includes the mo- tion space location of the target. Only in the uniform condition are the live-fly and dead-fly conditions truly symmetrical. Even in that case, Rosenholtz noted that the world around the subject is stationary, perhaps making it harder to detect stationary targets than moving targets. Regardless of how one understands the asymmetry between live- and dead-fly conditions, it is interesting to ask how that asymmetry is influenced by simulated motion of the observer, which is the purpose of Experiment 2.

\section{EXPERIMENT 2 \\ Dead Flies in Optic Flow Fields}

Given that Experiment 1 confirmed that it is easier to search for a moving item in a field of stationary items than vice versa, the question immediately arises as to how stationary and moving ought to be defined. Are these items stationary and moving in the world or on the retina? When observers move, the images of stationary objects move on the retina, and it is possible for an object that is moving in the world to have a stationary image on the retina. Does visual search take observer motion into account?

In order to test this, we simulated motion using three different types of flow fields: radial, random, and deformation. The radial flow field simulated observer motion toward a stationary scene. Radial expansion occurs as all of the circles move outward from a single point, the "Focus of Expansion" (Gibson, 1950). The stationary target in this case is a circle that is stationary on the computer monitor. As an analogy, think of a dead fly caught on a windshield. The fly is moving relative to the world outside the car, but its image on the driver's retina is stationary. Stationary objects in the world outside the car have images that move in a radial pattern on the observer's retina. This stimulus could, in principal, reverse the asymmetry seen in Experiment 1. If the image is interpreted as a flow field, then a target that is moving in the world is stationary in the image while distractors that are stationary in the world, move in the image.

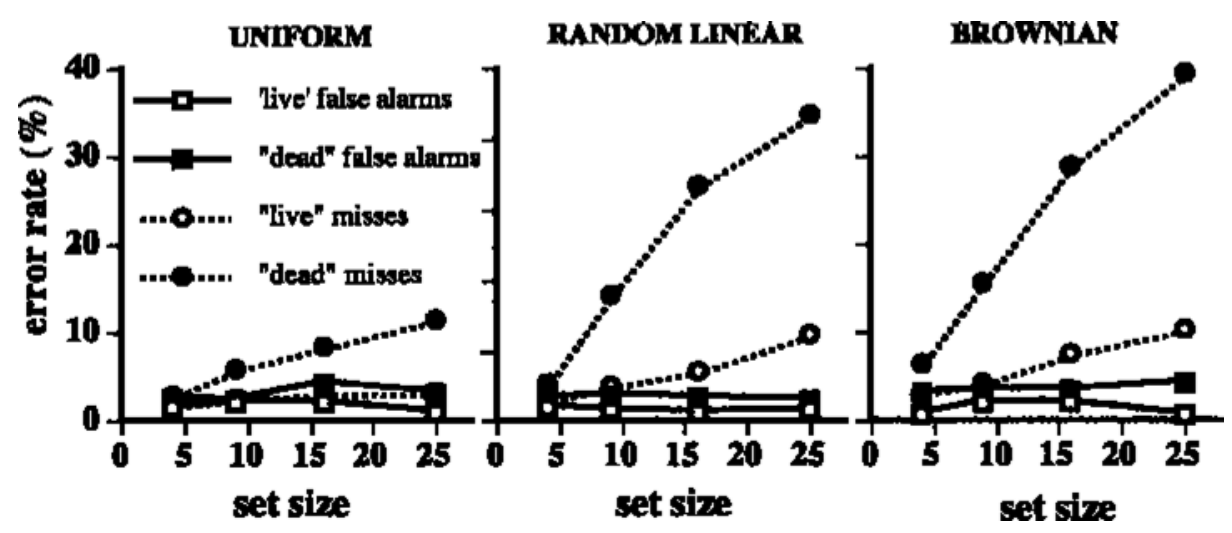

Figure 3. Error rates in Experiment 1. Each graph shows the error rate as a function of set size in Experiment 1 for the three different motion conditions, averaged across the 16 subjects. Squares indicate false alarms (when the subject incorrectly indicated the target was present), and circles indicate misses (when the subject incorrectly indicated the target was absent). Open symbols show the live-fly conditions, and filled symbols show the dead-fly conditions. 
Two control flow fields were tested for comparison with the radial condition. The deformation flow field is similar to the radial flow field, but the direction of the vertical component of motion has been reversed so that the pattern of motion appears to be moving inward vertically while expanding out horizontally. Throughout most of the image, the spatial gradients of motion for the radial and deformation flow patterns have the same magnitude. In other words, as you move from one position to the next on the image, the amount of change in the direction of motion of the elements is the same for deformation as it is for radial. Thus, the local properties of the deformation flow field are the same as those of the radial flow field. However, the global properties of the two flow fields are quite different. Whereas the radial pattern of flow is consistent with observer motion through a stationary scene, the deformation flow pattern is not.

The random flow field served as an example of an unstructured flow field. This pattern of flow was constructed with the same motion vectors as the radial flow field, but here the starting points of the motions were scrambled. So, the random flow field has the same motion vectors as the other two flow fields, but both the local and global properties differ. The random condition is similar to, but not identical to, the random linear condition of Experiment 1.

If search for motion targets is based on motion in the world rather than in the image, we might expect the radial condition produce efficient search, while the other two tasks produce inefficient search. If local image mo- tion is critical, the radial and deformation conditions might be expected to produce similar results. The random condition would be expected to produce inefficient search in either case.

\section{Method}

The experimental setup and task were similar to those in Experiment 1 . There were three different types of flow fields tested: radial, deformation, and random, as diagrammed in Figure 4. For each flow field, the observers were asked to detect the item whose image was stationary on the screen (as in the dead-fly conditions of Experiment 1). The radial flow field simulates observer motion toward a group of circular objects that range in distance between 400 and $1,000 \mathrm{~cm}$ from the observer and whose simulated sizes range from 3.0 to $10.0 \mathrm{~cm}$ in radius. Thus, the distractor radius ranged in size from $0.17^{\circ}$ to $1.43^{\circ}$. The target was a circle that had a radius chosen at random between $0.45^{\circ}$ and $1.15^{\circ}$. Target and distractors were initially distributed in a $20^{\circ} \times 20^{\circ}$ window as was described in Experiment 1. In each trial, the simulated direction of motion of the observer was chosen at random to be toward the screen and intersecting it within a $10^{\circ} \times 10^{\circ}$ square centered in the middle of the screen. Thus, all the distractors would move radially outward from this point of intersection. The simulated speed of the observer was $200 \mathrm{~cm} / \mathrm{sec}$. Thus, at the beginning of each trial, the average speed of the distractors at the intermediate distance to the observer $(700 \mathrm{~cm})$ was about $1.4^{\circ} / \mathrm{sec}$. The size of the distractors did not change over the course of a trial.

The deformation flow field was constructed in the same way as the radial flow field, except that the vertical component of motion for each of the distractors was inverted. Thus, the field expanded outward horizontally, while contracting vertically, giving a nonrigid appearance. The initial position of the distractors was within a $20^{\circ}$ $\times 30^{\circ}$ window. The increase in window height allowed the circles
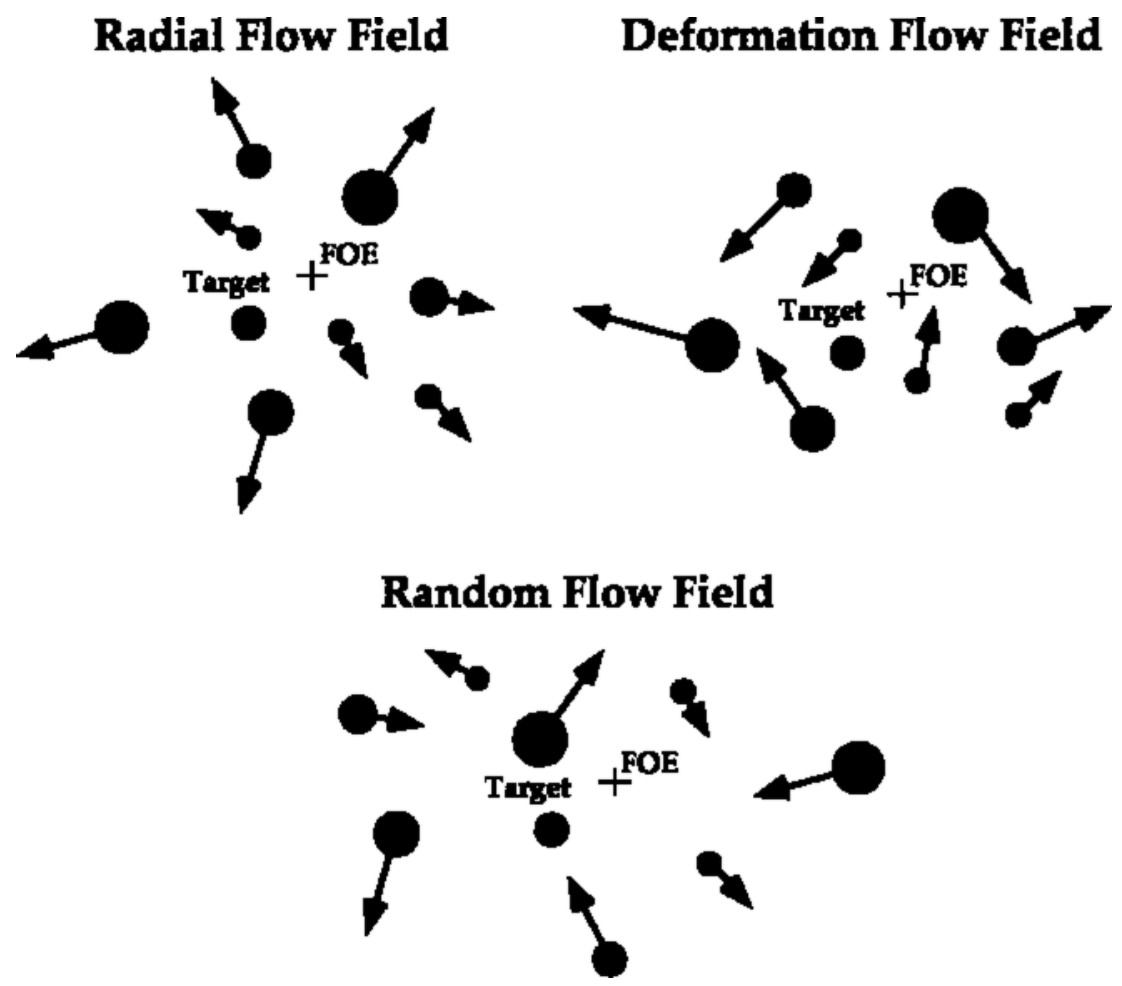

Figure 4. Stimulus conditions for Experiment 2. Arrows indicate the direction of motion of each circle. The FOE is indicated by a cross, which was not present on the display. 
to move inward toward the horizontal midline without becoming too crowded.

The random flow field used the same velocities as the radial flow field, but the initial starting position of each item was scrambled. Thus, the velocity vectors were identical to those in the radial flow field, but there was no ordered spatial pattern.

Twenty-one subjects participated in this experiment.

\section{Results}

Figure 5 shows the results for each of the three flow fields tested. Figure 5A shows results for target present trials, and Figure 5B shows the results for target absent trials. Average slopes, given in Table 2, in all conditions of this experiment are greater than zero (one-tailed $t$ tests, $p<.0001$ ), indicating that none of these conditions was the equivalent of a search for a moving target among stationary distractors. The flow and deformation conditions are significantly more efficient than the random condition (Fisher's PLSD post hoc comparisons, $p<.05$ ). As in Experiment 1, there were a large number of errors. These increase significantly with set size [ANOVA: $F(3,63)=$ $80.9, p<.0001]$, indicating that the slopes in Figure 5 underestimate the actual difficulty of the search. Error rates (shown in Figure 6) do not differ significantly as a function of condition [ANOVA: $F(2,42)=2.3, p=.12$ ].

The flow and deformation conditions produced slopes that are not significantly different from the dead target version of the uniform motion condition of Experiment 1 (all unpaired $t$ tests, $p>$.1). The random condition of Experiment 2 was less efficient than the uniform motion condition of Experiment 1 (unpaired $t$ tests, $p<.005$ for target present and target absent slopes). The flow and deformation conditions were significantly more efficient than the dead target version of the random motion condition of Experiment 1 (all unpaired $t$ tests, $p<.01$ ). The random condition of Experiment 2 was not statistically
Table 2

Slopes for Each of the RT $\times$ Set Size Functions for Experiment 2

\begin{tabular}{crcc}
\hline & Flow & Deformation & Random \\
\hline Present & 6.5 & 6.5 & 10.7 \\
Absent & 13.6 & 8.2 & 19.4 \\
\hline
\end{tabular}

Note-The slopes are given in milliseconds per item. (See Figure 5 for graphs of the slopes.)

different from the random motion condition of Experiment 1 (unpaired $t$ tests, $p>.05$ for target present and absent slopes).

\section{Discussion}

The results of Experiment 2 give no support to the hypothesis that radial flow fields have a special status in visual search for motion. If the radial flow field had been interpreted as representing a stationary array of dots viewed by a moving observer, then the target item, stationary in the display, should have been interpreted as a moving item in the world. The search should have been an efficient search for a moving target among stationary distractors. In Experiment 1, all three versions of such a search produced slopes that were not significantly different from zero. In Experiment 2, the flow condition produced slopes that were significantly different from zero and were not significantly different from control conditions for stationary targets among moving distractors. The relevant controls, in this case, were the deformation condition of Experiment 2 and the uniform condition of Experiment 1. The deformation condition had all of the same motion vectors as the flow condition but did not allow for an interpretation of observer motion. Both of these stimuli contained distractor motions in many directions. Given that distractor heterogeneity is supposed

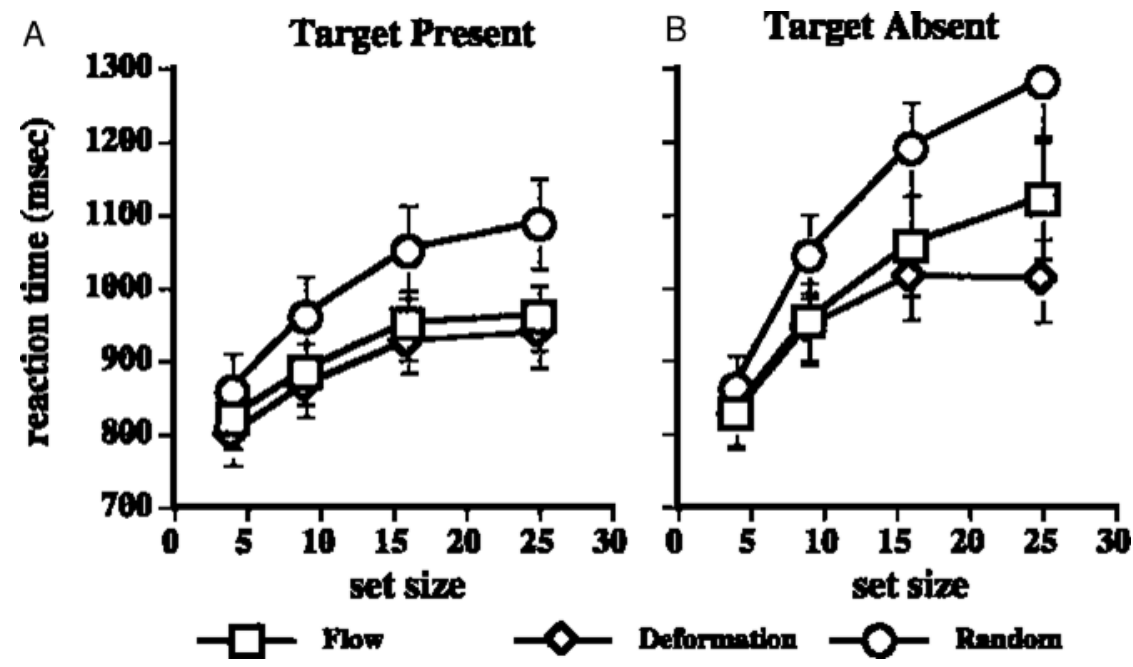

Figure 5. Results of Experiment 2. Each graph shows the RT $\times$ set size functions for the three conditions in Experiment 2, averaged across 21 subjects. The left graph shows results for target present trials, and the right graph shows results for target absent trials. Squares, diamonds, and circles represent the flow, deformation, and random motion conditions, respectively. Error bars are $\pm 1 S E$. 

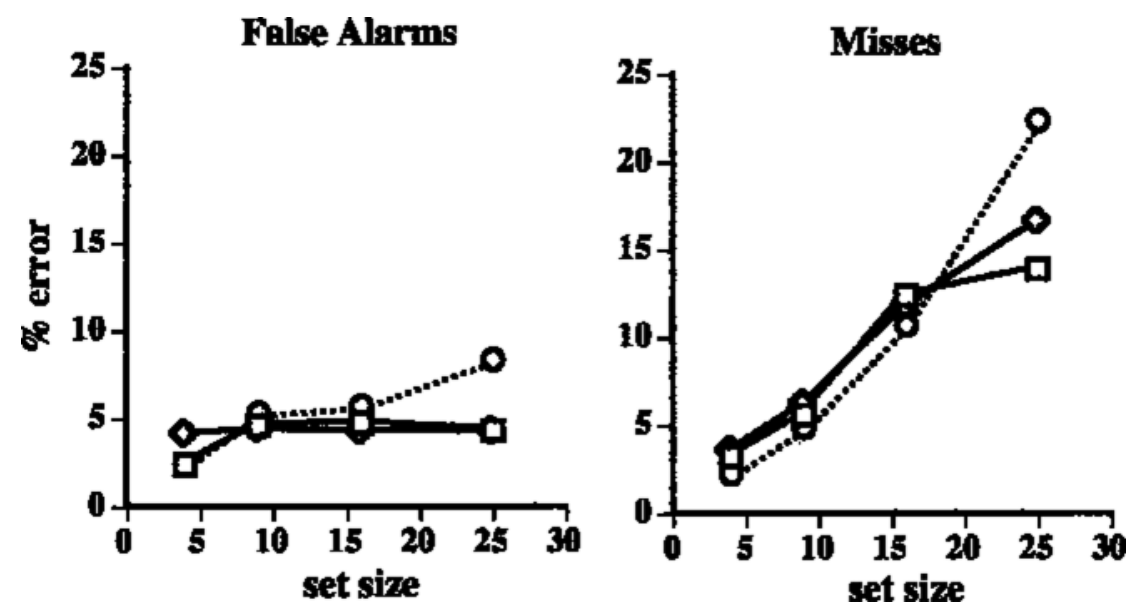

Figure 6. Error rates in Experiment 2. Each graph shows error rate $\times$ set size functions for the three conditions in Experiment 2, averaged across 21 subjects. Squares, diamonds, and circles represent the flow, deformation, and random motion conditions, respectively. Open diamonds represent the deformation condition.

to make search less efficient, why were these conditions no less efficient than the uniform condition of Experiment 1 ? Why were they more efficient than the random motion conditions of the two experiments?

The critical factor is most likely the relatively slow change in direction of motion across the image. Local patches of flow and deformation displays do not differ substantially from those of the uniform flow field of Experiment 1 . In very analogous experiments, Moraglia (1989) and Nothdurft (1993) have shown that local differences in orientation determine search efficiency. Thus, for example, a vertical line target can be found easily if its local neighbors are horizontal, even if there are other vertical lines elsewhere in the display. Nothdurft illustrated this principle for local variation in color and motion (see also Treisman, 1982). Some of Nothdurft's motion displays are similar in spirit to our deformation condition. The results of our Experiment 2 are consistent with the view that the local disruption of the motion field caused by a stationary item can attract attention, albeit imperfectly, but that there is nothing special about the radial pattern of optic flow.

We do not think that this result is an artifact of the specific flow pattern used in Experiment 2. In a number of other pilot studies, we failed to find any flow pattern that reversed the asymmetry of Experiment 1 . However, we did find that moving objects within an optic flow field could be found efficiently if their motion differed sufficiently in angle or speed from the rest of the flow field (Royden, Wolfe, Konstantinova, \& Hildreth, 1996).

\section{GENERAL DISCUSSION}

Although there is an unequivocal asymmetry in search efficiency for moving versus stationary targets, the difference in slopes between the two situations might not seem as dramatic as would be expected. In the clearest of search asymmetries, the more efficient search would yield slopes near zero, whereas the less efficient search would yield slopes in the classic "serial search" range of 20-30 msec/item for target present trials and about twice that for target absent. The slopes for the less efficient dead versions of Experiment 1 are not this steep. In the case of the random and brownian conditions, it is clear that this is a simple case of a speed-accuracy tradeoff (Ruthruff, 1996). The subjects produced very high rates of errors that increased with set size. If the stimuli had been of longer duration and had we pushed the subjects to greater accuracy, the slopes would have been markedly steeper. Other factors may have contributed to the fairly efficient search in the uniform motion condition. One possibility is that the uniformity of motion among the distractors made it easier for the subjects to group them into a single object and reject them as a group than it would have been for the case when all the distractors moved in different directions (Driver, 1992; Duncan, 1995; Grossberg, Mingolla, \& Ross, 1994; Pashler, 1987; Treisman, 1982; Yantis, 1992). An induced motion effect (Dunker, 1938; Gogel, 1979) might also have contributed to relatively efficient search. That is, the moving distractors could have induced the perception of target motion in the opposite direction. It is known that targets moving in a direction opposite to that of the distractors are found efficiently (Dick et al., 1987; Nakayama \& Silverman, 1986), so it is possible that a target with induced motion in the opposite direction from the distractors might also be found reasonably efficiently. A related possibility is that the uniform motion of the distractors may have induced tracking eye movements. In this situation, the retinal stimulus becomes like the live-fly condition, in which the images of the distractors are stationary on the retina, and the image of the target is moving. If this were the 
case, however, it is unclear why the search efficiency for the uniform motion condition was not as high for the dead-fly condition as it was for the live-fly condition.

An optic flow or observer motion account could provide an alternative explanation for the relative ease of finding stationary targets among uniformly moving distractors. Perhaps the brain discounts motion that could be due to lateral observer motion. The uniform motion stimulus is consistent with an observer moving in a direction opposite to that of the distractors. The target in this case would be moving at the same speed and direction as the observer, and thus its image would be stationary on the retina. If the brain has evolved an efficient mechanism to find moving stimuli in the world, it might do so by discounting any retinal motion that could be generated by observer motion during search for a target.

Of course, this optic flow account of the uniform condition would be more compelling if there had been good evidence for a role for optic flow in the flow condition of Experiment 2. Should we conclude that visual search processes cannot discount the motion produced by observer motion? There is little previous data on search within an optic flow background. Two studies examined search for an expanding target amid contracting or deforming distractors and vice versa (Braddick \& Holliday, 1991; Takeuchi, 1997), but in these experiments each local item expanded or contracted. This is not equivalent to the global expansion pattern generated by observer motion. Perhaps more relevant is the fact that recent experiments have shown that judgments of heading change little when subjects are attending another, simultaneous task (Royden \& Hildreth, 1999). This suggests that the processing of optic flow fields did not compete for attentional resources. Either flow fields were analyzed "pre-attentively" prior to an attentional bottleneck or the flow fields were analyzed in some pathway separate from that used for the attention-demanding task.

One could take the results of the present Experiment 2 to be evidence for the second option. Simulated observer motion failed to reverse the search asymmetry because the calculation of observer motion was separate from the processes involved in visual search. However, there is an alternative that cannot be ruled out in these experiments. It might be that our simulated observer motion was simply not compelling enough to be discounted. The stimulus was not particularly large nor was the stimulus particularly rich or natural. It is possible that different results would be obtained with a different version of the flow condition (e.g., a virtual reality stimulus). The answer to this question awaits a different series of experiments.

Three conclusions follow from the present experiments: (1) We confirm the previously reported search asymmetry that renders search for motion much more efficient than search for its absence. (2) We provide support for the hypothesis that search efficiency in feature search is based more on local differences than on the global properties of the display. Finally, (3) we can reject the hypothesis that the visual system treats all radial flow fields differ- ently from other structured motion fields in visual search experiments.

\section{REFERENCES}

Braddick, O. J., \& Holliday, I. E. (1991). Serial search for targets defined by divergence or deformation of optic flow. Perception, 20, 345-354.

Bravo, M., \& Blake, R. (1990). Preattentive vision and perceptual groups. Perception, 19, 515-522.

Cavanagh, P., Arguin, M., \& Treisman, A. (1990). Effect of surface medium on visual search for orientation and size features. Journal of Experimental Psychology: Human Perception \& Performance, 16, 479-492.

Dick, M., Ullman, S., \& Sagi, D. (1987). Parallel and serial processes in motion detection. Science, 237, 400-402.

DrIVER, J. (1992). Motion coherence and conjunction search: Implications for guided search theory. Perception \& Psychophysics, 51, 79-85.

DuncAN, J. (1995). Target and nontarget grouping in visual search. Perception \& Psychophysics, 57, 117-120.

Duncan, J., \& Humphreys, G. W. (1989). Visual search and stimulus similarity. Psychological Review, 96, 433-458.

DUNKER, D. (1938). Induced motion. In W. D. Ellis (Ed.), A sourcebook of Gestalt psychology (pp. 161-172). London: Routledge \& Kegan Paul.

Foster, D. H., \& WARD, P. A. (1991). Asymmetries in oriented-line detection indicate two orthogonal filters in early vision. Proceedings of the Royal Society of London: Series B, 243, 75-81.

GiBson, J. J. (1950). The perception of the visual world. Boston: Houghton Mifflin.

GogeL, W. C. (1979). Induced motion is a function of the speed of the moving object, assessed by means of two methods. Perception, 8 , 255-262.

Grossberg, S., Mingolla, E., \& Ross, W. D. (1994). A neural theory of attentive visual search: Interactions of boundary, surface, spatial and object representations. Psychological Review, 101, 470-489.

Gurnsey, R., Humphrey, G. K., \& Kapitan, P. (1992). Parallel discrimination of subjective contours defined by offset gratings. Perception \& Psychophysics, 52, 263-276.

IV RY, R. B., \& CoHEN, A. (1992). Asymmetry in visual search for targets defined by differences in movement speed. Journal of Experimental Psychology: Human Perception \& Performance, 18, 1045-1057.

MCLeOD, P., DrIVER, J., \& CRISP, J. (1988). Visual search for conjunctions of movement and form is parallel. Nature, 332, 154-155.

Moore, C. M., \& Wolfe, J. M. (2000). Getting beyond the serial/parallel debate in visual search. In K. Shapiro (Ed.), The limits of attention: Temporal constraints on human information processing. Oxford: Oxford University Press.

Moraglia, G. (1989). Display organization and the detection of horizontal line segments. Perception \& Psychophysics, 45, 265-272.

Nakayama, K., \& Silverman, G. H. (1986). Serial and parallel processing of visual feature conjunctions. Nature, 320, 264-265.

NothduRfT, H.-C. (1993). The role of features in preattentive vision: Comparison of orientation, motion and color cues. Vision Research, 33, 1937-1958.

Pashler, H. (1987). Detecting conjunctions of color and form: Reassessing the serial search hypothesis. Perception \& Psychophysics, 41, 191-201.

Rosenholtz, R. (2001). Search asymmetries? What search asymmetries? Perception \& Psychophysics, 63, 476-489.

Royden, C. S., \& Hildreth, E. C. (1999). Differential effects of shared attention on perception of heading and 3-D object motion. Perception \& Psychophysics, 61, 120-133.

Royden, C. S., Wolfe, J. M., Konstantinova, E., \& Hildreth, E. C. (1996). Search for a moving object by a moving observer [Abstract]. Investigative Ophthalmology \& Visual Science, 37, S299.

RuthrufF, E. (1996). A test of the deadline model for speed-accuracy tradeoffs. Perception \& Psychophysics, 58, 56-64.

TAKEUCHI, T. (1997). Visual search of expansion and contraction. $V i$ sion Research, 37, 2083-2090. 
Townsend, J. T. (1990). Serial and parallel processing: Sometimes they look like Tweedledum and Tweedledee but they can (and should) be distinguished. Psychological Science, 1, 46-54.

Treisman, A. (1982). Perceptual grouping and attention in visual search for features and for objects. Journal of Experimental Psychology: Human Perception \& Performance, 8, 194-214.

Treisman, A., \& Gelade, G. (1980). A feature-integration theory of attention. Cognitive Psychology, 12, 97-136.

Treisman, A., \& Gormican, S. (1988). Feature analysis in early vision: Evidence from search asymmetries. Psychological Review, 95, 15-48.

Treisman, A., \& Souther, J. (1985). Search asymmetry: A diagnostic for preattentive processing of separable features. Journal of Experimental Psychology: General, 114, 285-310.
Verghese, P., \& Pelli, D. G. (1992). The information capacity of visual attention. Vision Research, 32, 983-995.

Von Grünau, M., \& Dubé, S. (1994). Visual search asymmetry for viewing direction. Perception \& Psychophysics, 56, 211-220.

Wolfe, J. M. (1998). Visual search. In H. Pashler (Ed.), Attention (pp. 13-74). Hove, U.K.: Psychology Press.

YANTIS, S. (1992). Multielement visual tracking: Attention and perceptual organization. Cognitive Psychology, 24, 295-340.

(Manuscript received April 11, 2000; revision accepted for publication September 21, 2000.) 